\title{
Why some Emergency Department patients with admission orders do not get quickly transferred to the inpatients?
}

\author{
Butt T ${ }^{* *}$, Qureshi $\mathrm{M}^{2}$, Alshudukhi $\mathrm{A}^{3}$, Alkishi $\mathrm{A}^{3}$, Burns $\mathrm{S}^{4}$, Alaqeel $\mathrm{S}^{5}$, Alrashidi $\mathrm{A}^{5}$ and Alali $\mathrm{A}^{2}$ \\ ${ }^{1}$ Chairman, Department of Emergency Medicine, King Faisal Specialist Hospital and Research Centre (KFSH\&RC)-Madinah, KSA \\ ${ }^{2}$ Consultant, Emergency Medicine, KFSH\&RC, Riyadh, KSA \\ ${ }^{3}$ Emergency Medicine Resident, KFSH\&RC, Riyadh, KSAAssistant Head Nurse, KFSH\&RC, Riyadh, KSA \\ ${ }^{4}$ Medical Student, College of Medicine, Al Maarefa University, Riyadh, KSA
}

\begin{abstract}
The admitted Emergency Department (ED) patients should be disposed to an appropriate clinical inpatient bed based on validated clinical criteria. The patients should not be held unnecessarily in ED once the decision to admit has been made. The mortality of these admitted patients increases proportionately with the length of stay in ED. Patient movement from ED should be based on accredited criteria based on Hospital resources. Lack of use of validated criteria can lead to unnecessary delay and ED patient boarding. Admitted patients from the ED who are denied access to inpatient units may continue to have abnormal vital signs for several days.
\end{abstract}

\section{Introduction}

The disposition of admitted patients in the Emergency Department (ED) should happen without any significant delay. Once the initial resuscitation and stabilization has occurred, admitted patients should be transferred to an appropriate clinical setting [1]. A delay in transferring these patients causes ED boarding, which stacks up clinical risk [2]. The boarded patients' mortality increases significantly with every hour of stay in ED, including those patients who need admission to intensive care [3].

Most hospitals will use a standardized criterion to transfer patients out of the ED to an appropriate unit. Our institution uses measures adopted from the hospitals" "Rapid Response Team" (RRT) activation criteria (Figure 1) which are intended for hospital inpatients. RRT has been successfully used to identify inpatients who require urgent interventions to prevent clinical deterioration [1-3]. These "advanced RRT criteria" (ARRT) comprises of patients' vitals, clinical presentations, laboratory parameters and health care worker clinical gestalt.

The reliability of RRT activation and early intervention with management of admitted patients in the hospital has been clearly documented with reduction in mortality [2]. However, the use of these criteria to determine the suitability of admitted ED patients for inpatient ward transfer is not a common practice. Our institution uses the ARRT criteria to determine the suitability of ED boarded patients for inpatient ward, intensive care unit (ICU) or coronary care unit (CCU) transfers. The patients who do not fit under these criteria are considered unfit and remain in the ED as boarded patients.

We wanted to find the common reason for an ED patient with admission orders to continue to stay in the ED and being unfit for transfer to inpatient bed.

\section{Methods \& statistical analysis}

The cohort of ED patients with admission orders who were not considered fit for transfer to the inpatients were studied over a period of three months. Patients who were fit to be transferred but were boarded in ED due to the lack of availability of an appropriate inpatient bed were excluded.

List of ED patient with admission orders was obtained daily from the hospital electronic system (ICIS). The ED charge nurses' list of admitted patients who were considered unfit for inpatient transfer was also obtained daily during the study period. Each patients' electronic medical record was reviewed in detail to corroborate the unfit reasons mentioned in the manual list.

We collected the data of our selected patients including reason for being unfit for ward transfer, demographics, code status, admitting service, reason for admission, duration of ED boarding due to being unfit and disposition.

Descriptive statistics for the continuous variables were reported as mean \pm standard deviation and categorical variables were interpreted as frequencies and percentages. The categorical variables were compared by Chi-square test and the continuous variables were compared by Student's t-test or ANOVA. Univariate and multivariate logistic regression was used to identify the independent variables that contribute significantly to major outcomes of this study. The level of statistical significance is set at $\mathrm{p}<0.05$.

${ }^{\star}$ Correspondence to: Taimur S. Butt, Chairman, Department of Emergency Medicine, King Faisal Specialist Hospital \& Research Centre- Madinah, KSA, E-mail: tbutt@kfshrc.edu.sa

Received: May 21, 2020; Accepted: June 15, 2020; Published: June 18, 2020 
Adult

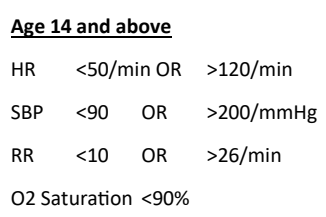

O2 Saturation $<90 \%$

Capillary refill $>4 \mathrm{sec}$

1-12 months

HR $<80 / \mathrm{min}$ OR $\quad>200 / \mathrm{min}$

SBP $\quad<70 / \mathrm{mmHg}$

RR $<20$ OR $>80 / \mathrm{min}$

1-14 years

HR $<70 / \mathrm{min}$ OR $>180 / \mathrm{min}$

SBP $\quad<90 / \mathrm{mmHg}$

RR $\quad<15$ OR $>60 / \mathrm{min}$

Figure 1. Rapid response team (RRT) activation criteria

Patients identity was protected, and consent was not required as this was an observational study without any interventions.

\section{Results}

During the study period of three months, 119 patients met the inclusion criteria for our study. 55 (46\%) of patients were females with a mean age of 45.1 years ( 0.1 - 93 CI (Confidence Interval) 95\%).

50 (42\%) patients were admitted under the Department of Medicine. Transplant Center had 16 (13\%), Medical Oncology 14 (12\%), Department of Surgery had 4 (3\%) and Department of Pediatrics had 5 (4\%) patients. Four (3\%) patients were not accepted by inpatient ward due to non-availability of a monitored bed. Two patients (2\%) were delayed because of a need for clinical reassessment in the ED by the admitting service. One (1\%) patient stayed in the ED as the admitting service refused authorize transfer to an available bed (Table 1).

In 43 (36\%) patients, tachypnea was the reason for being unfit for medical floor transfer. Isolated tachypnea was present in 28 (24\%) patients. Tachycardia was documented as a reason in $19(16 \%)$ patients. Ten patients $(8 \%)$ had tachycardia as the only reason for being unfit. Two (2\%) patients were reported to be unfit due to bradycardia. 13 (11\%) patients were unfit due to hypotension. Two (2\%) patients were considered unfit due to persistent seizure activity (Tables 2 and 3).

Electrolyte abnormalities resulted in delay in medical ward transfer in $11(9 \%)$ patients. Hyponatremia reported in 7 (6\%) patients was the most common electrolyte abnormality. Hypokalemia, hyperkalemia hypoglycemia and hypernatremia were each present in 1 (1\%) patient.

Code status of 106 (89\%) patients was full code, while 7 (6\%) were "Do Not Attempt Resuscitation (DNAR)" status at the time of admission and $6(5 \%)$ were DNAR with interventions. 8 (7\%) patients' code status was changed before moving to the medical floor to DNAR while boarding in the ED (Table 4). The mean boarding time was 31.8 hours (2 - 162 hours CI 95\%) (Table 5).
97 (81\%) of the ED boarded unfit patients were eventually sent to the inpatient ward. 17 (14\%) were sent to an intensive care or coronary care unit while 5 (4\%) patients were discharged home.

\section{Discussion}

Timely transfer of admitted ED patients to a clinical area appropriate to the needed level of care i.e., intensive care, coronary care, operating room, or med-surgical floor will decrease patient risk and improve quality of care (Figure 2). Inpatient level of care is determinted by the treating physician expertise, available resources, total nursing hours and nursing skill mix [4].

RRT activation criteria have been used by hospitals for several years as an aid for recognizing clinical deterioration of inpatients. Our institution has a well-established inpatient RRT that operated 24/7. This was developed following the recommendations from the Institute of Healthcare Improvement Campaign to manage inpatients [5]. The RRT criteria are validated for early intervention during inpatient deterioration.

Table 1. Admitting service

\begin{tabular}{|l|c|c|c|c|}
\hline Service & Frequency & Percent & $\begin{array}{c}\text { Cumulative } \\
\text { Frequency }\end{array}$ & $\begin{array}{c}\text { Cumulative } \\
\text { Percent }\end{array}$ \\
\hline Cardiology & 6 & 5.04 & 6 & 5.04 \\
\hline Dept. of Surgery & 4 & 3.36 & 10 & 8.40 \\
\hline Hem/Oncology & 1 & 0.85 & 11 & 9.25 \\
\hline ICU & 1 & 0.85 & 12 & 10.10 \\
\hline Department of Medicine & 50 & 42.01 & 62 & 52.11 \\
\hline Organ Transplant & 16 & 13.44 & 78 & 65.55 \\
\hline Medical Genetics & 3 & 2.52 & 81 & 68.07 \\
\hline Medical oncology & 14 & 11.76 & 95 & 79.83 \\
\hline Neurology & 7 & 5.88 & 102 & 85.71 \\
\hline OB/GYN & 2 & 1.70 & 104 & 87.41 \\
\hline PICU & 1 & 0.85 & 105 & 88.26 \\
\hline Pediatric oncology & 2 & 1.70 & 107 & 89.96 \\
\hline Pediatric surgery & 2 & 1.70 & 109 & 91.66 \\
\hline Department of Pediatrics & 5 & 4.20 & 114 & 95.86 \\
\hline Urology & 2 & 1.70 & 116 & 97.56 \\
\hline Neurosurgery & 1 & 0.85 & 117 & 98.41 \\
\hline Orthopedics & 1 & 0.85 & 118 & 99.26 \\
\hline Frequency Missing = & & & & \\
\hline
\end{tabular}

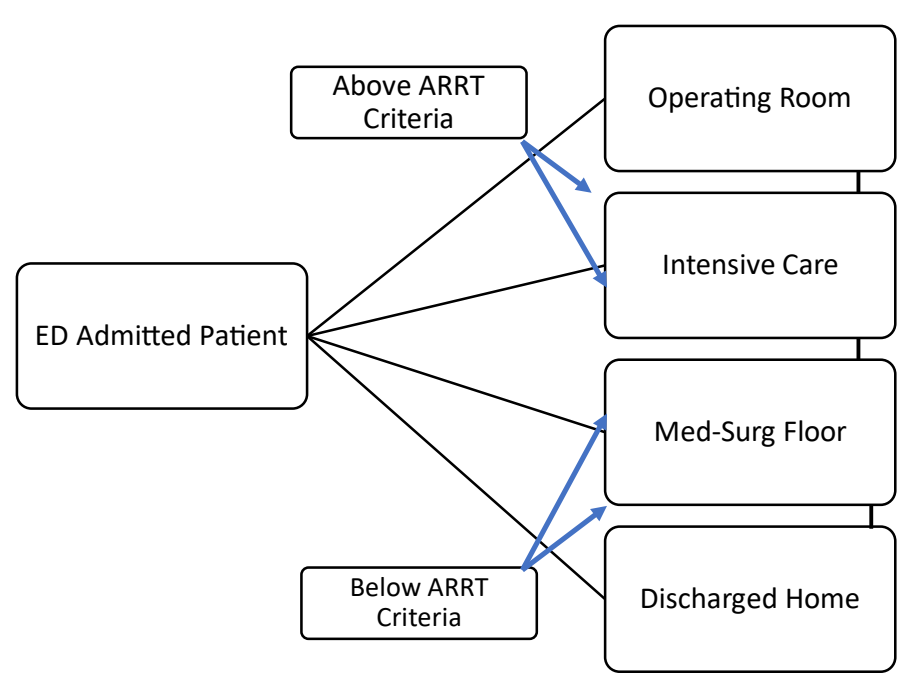

Figure 2. Flow of ED admitted patients 
Table 2. Reasons to be Unfit: RRT criteria

\begin{tabular}{|l|c|c|c|c|}
\hline Reasons & Frequency & Percent & $\begin{array}{c}\text { Cumulative } \\
\text { Frequency }\end{array}$ & $\begin{array}{c}\text { Cumulative } \\
\text { Percent }\end{array}$ \\
\hline Bradycardia & 1 & 0.84 & 1 & 0.84 \\
\hline Hypoxia & 1 & 0.84 & 2 & 1.68 \\
\hline Multiple seizures & 2 & 1.68 & 4 & 3.36 \\
\hline Hypertension & 4 & 3.36 & 8 & 6.72 \\
\hline Hypotension & 11 & 9.24 & 19 & 15.96 \\
\hline Tachycardia & 12 & 10.08 & 31 & 26.04 \\
\hline Tachypnea & 22 & 18.48 & 53 & 44.52 \\
\hline Multiple RRT criteria & 32 & 26.89 & 85 & 71.42 \\
\hline
\end{tabular}

Table 3. Reasons to be unfit

\begin{tabular}{|l|c|c|c|c|}
\hline Reasons & Frequency & Percent & $\begin{array}{c}\text { Cumulative } \\
\text { Frequency }\end{array}$ & $\begin{array}{c}\text { Cumulative } \\
\text { Percent }\end{array}$ \\
\hline Physician Decision & 2 & 1.68 & 2 & 1.68 \\
\hline GCS 13 & 1 & 0.84 & 3 & 5.88 \\
\hline High CO2 & 1 & 0.84 & 4 & 8.40 \\
\hline Electrolyte Imbalance & 12 & 10.08 & 16 & 13.44 \\
\hline ICU follow up & 5 & 4.20 & 21 & 17.64 \\
\hline Labetalol infusion & 1 & 0.84 & 22 & 18.48 \\
\hline Needs Monitor Bed & 4 & 3.36 & 26 & 21.84 \\
\hline Endoscopy & 1 & 0.84 & 27 & 22.68 \\
\hline Intubated & 4 & 3.36 & 31 & 26.04 \\
\hline Low Hemoglobin & 2 & 1.68 & 33 & 27.72 \\
\hline Patient on BIPAP & 1 & 0.84 & 34 & 28.56 \\
\hline
\end{tabular}

Table 4. Code status on admission

\begin{tabular}{|l|c|c|c|c|}
\hline Code Status & Frequency & Percent & $\begin{array}{c}\text { Cumulative } \\
\text { Frequency }\end{array}$ & $\begin{array}{c}\text { Cumulative } \\
\text { Percent }\end{array}$ \\
\hline DNAR & 7 & 5.88 & 7 & 5.88 \\
\hline DNAR with intervention & 6 & 5.04 & 13 & 10.92 \\
\hline Full & 106 & 89.08 & 119 & 100.00 \\
\hline
\end{tabular}

Table 5. Boarding hours

\begin{tabular}{|c|c|c|c|c|}
\hline $\mathbf{N}$ & Mean & Std Dev & Minimum & Maximum \\
\hline 91 & 31.8 & 31.3 & 2.0 & 162.0 \\
\hline
\end{tabular}

In our institution, the RRT does not cover admitted patients while boarding in the ED. Any clinical deterioration in these patients is the responsibility of the admitting service and the ED team. However, our inpatient units measure ED patients' stability and suitability for their ward or the unit based on the ARRT criteria. Incident reports are generated by the inpatient teams if ED fails to comply with the hospital guidelines and inadvertently transfers admitted patients to inappropriate destination. During our study period no such incidents were reported, indicating ED staff strict compliance with the institutional guidelines.

The RRT criteria include patient vital signs (heart rate, systolic blood pressure, respiratory rate, or oxygen saturation). In addition, the ARRT criteria include clinical signs like "significant bleeding", deterioration in the Glasgow Coma Scale (GCS), seizure activity etc., laboratory values and clinical gestalt (Figure 1). Our study results indicate that use of ARRT criteria for ED admissions resulted in delay of patient transfers to the inpatient units with resultant increase in ED boarding of admitted patients.

It is quite conceivable that the patients who are being admitted for inpatient care from an ED are likely to have abnormal vital signs and or lab results. This was obviously noted in our study group. Based upon the resource allocation to each clinical area, patients with critical condition should be admitted to the ICU/CCU and the others to a medical-surgical ward. Many institutions have intermediate care unit with telemetry capabilities and nurse-patient ratios between those in ICU and medical floor. Non-availability of a variety of inpatient beds and a gap between the intensive care and ward acceptance criteria may result in excessive ED boarding [6]. Even though, most of our patients were eventually transferred to the inpatient units, but few patients were discharged home without reaching an inpatient area.

In the United Kingdom (UK), the National Health Service (NHS) uses the National Early Warning Score (NEWS) based on the clinical severity, as the criteria for allocating an inpatient bed for ED admissions. Level 0 is a standard inpatient bed and Level 4 is an ICU bed. NEWS is like RRT criteria but is applied both in the ED and inpatient settings. Success of any system is based upon the accuracy and reproducibility of data elements [7].

At present, in the Kingdom of Saudi Arabia (KSA) RRT activation criteria with local and institutional modifications are being used with no agreed national criteria for deciding patients' clinical severity or disposition from the ED. Bed nomenclature also varies in different institutions. These can be standard inpatient wards, intermediate beds, transitional zones, step down units (SDUs) etc. SDU is defined as the level of care that is intermediate between standard ward and intensive care bed [7]. Some KSA hospitals have made intermediate medical care unit (IMCU), where patients destined for ICU can be managed for 24 hours before transfer to ICU. Standardized healthcare staff communication would also limit patient risk and delay in patient disposition $[8,9]$.

The main reason for access block in any ED is the out-flow problem with lack of inpatient beds and boarding of admitted patients in the ED [10]. Using strict inpatient acceptance criteria without clear outflow processes would result in excessive ED boarding as noted in our study. ICU acceptance criteria can become overly complex and may pose ethical challenges [11]. Some of our patient's code status was changed to incorporate these patients with abnormal vital signs and clinical parameters to a med-surgical floor. At times, improper code status designation or delayed change may prolong ED stay which may result in patient deterioration and risk of iatrogenic complications i.e., inappropriate intubation, bedsores, falls, and nosocomial infections. Rapid disposition of ED admissions based on standardized criteria will not only improve ED flow but will also minimize patient risk.

\section{Limitations}

Boarded patients' morbidity and mortality rates were not evaluated. The effect of these criteria on the ED waiting times, inpatient length of stay or patient outcomes was not measured.

\section{Conclusion}

Majority of the ED boarded patients have more than one abnormal vital sign from amongst the RRT criteria which impedes their inpatient transfer. Tachypnea was the most common individual justification for continued boarding in the ED and delay in patient transfer for many hours. In addition, several non-RRT criteria are being used by staff to determine patient stability during ward or ICU shifting. Development of clinical areas with continuous vital signs monitoring capabilities, advanced nursing skill sets and adequate resources in the inpatient units may improve ED admitted patients' flow and reduce boarding hours.

\section{References}

1. Stevens Jennifer P. Rapid response systems.

2. Michael B, Bernard S, Nguyen TV, Moore G, Anderson J (2004) Association between clinically abnormal observations and subsequent in-hospital mortality: a prospective study. Resuscitation 2: 137-141. 
Butt T (2020) Why some Emergency Department patients with admission orders do not get quickly transferred to the inpatients?

3. Meera P, Myles PS, Leach DS, Maclean AV (2002) Outcome of emergency department patients with delayed admission to an intensive care unit. Emergency Medicine 14: 50-57.

4. Thomas LA (2004) Nurse-patient ratios: a systematic review on the effects of nurse staffing on patient, nurse employee, and hospital outcomes. JONA: The Journal of Nursing Administration 34: 326-337.

5. Jeremy RB (2011) Reduction in hospital-wide mortality after implementation of a rapid response team: a long-term cohort study. Critical Care 15: R269.

6. Leora IH (2009) Dropping the baton: a qualitative analysis of failures during the transition from emergency department to inpatient care. Annals of Emergency Medicine 53: 701-710.
7. Mike J (2012) NEWSDIG: The national early warning score development and implementation group. Clinical Medicine 12: 501.

8. Meghan P, Wunsch H (2014) The role of stepdown beds in hospital care. American Journal of Respiratory and Critical Care Medicine 190: 1210-1216.

9. Joint Commission Resources (2004) Improving Care in the ICU. Joint Commission on Accreditation of Healthcare Organizations.

10. Daniel MF, Nagree Y, Sprivulis P (2005) Access block causes emergency department overcrowding and ambulance diversion in Perth, Western Australia. Emergency Medicine Journal 22: 351-354.

11. Joseph LN (2016) ICU admission, discharge, and triage guidelines: a framework to enhance clinical operations, development of institutional policies, and further research. Critical Care Medicine 44: 1553-1602.

Copyright: (C)2020 Butt T. This is an open-access article distributed under the terms of the Creative Commons Attribution License, which permits unrestricted use, distribution, and reproduction in any medium, provided the original author and source are credited. 